\title{
Decentralized Validation for Non-malicious Arbitrary Fault Tolerance in Paxos
}

\author{
Rodrigo R. Barbieri ${ }^{2}$, Enrique S. dos $\operatorname{Santos}^{1}$, Gustavo M. D. Vieira $^{1}$ \\ ${ }^{1}$ DComp - CCGT - UFSCar \\ Sorocaba, São Paulo, Brasil \\ enrique.santos@dcomp.sor.ufscar.br, gdvieira@ufscar.br \\ ${ }^{2}$ Flextronics Instituto de Tecnologia \\ Sorocaba, São Paulo, Brasil \\ rodrigo.barbieri2010@gmail.com
}

\begin{abstract}
Fault-tolerant distributed systems offer high reliability because even if faults in their components occur, they do not exhibit erroneous behavior. Depending on the fault model adopted, hardware and software errors that do not result in a process crashing are usually not tolerated. To tolerate these rather common failures the usual solution is to adopt a stronger fault model, such as the arbitrary or Byzantine fault model. Algorithms created for this fault model, however, are considerably more complex and require more system resources than the ones developed for less strict fault models. One approach to reach a middle ground is the non-malicious arbitrary fault model. This model assumes it is possible to detect and filter faults with a given probability, if these faults are not created with malicious intent, allowing the isolation and mapping of these faults to benign faults. In this paper we describe how we incremented an implementation of active replication in the non-malicious fault model with a basic type of distributed validation, where a deviation from the expected algorithm behavior will make a process crash. We experimentally evaluate this implementation using a fault injection framework showing that it is feasible to extend the concept of non-malicious failures beyond hardware failures.
\end{abstract}

\section{Introduction}

Fault-tolerant distributed systems offer high reliability because even if faults in their components occur, they do not exhibit erroneous behavior. Depending on the fault model adopted, common faults such as message loss and processes crashes have no effect on the distributed algorithm being run. However, hardware and software errors that do not result in a process crashing are usually not tolerated in the most used fault models. Considering the hardware, examples of these faults are memory corruption caused by spontaneous bitflips, disk corruption caused by media defects, message corruption due to interference, among others. Looking at the software, programmer error, operating system bugs and operator error can violate the assumptions of the target fault model.

To tolerate these rather common failures the usual solution is to adopt a stronger fault model that assumes any type of fault can occur. The arbitrary or Byzantine fault model [Lamport et al. 1982] is very well understood and in fact offers tolerance to a wide range of fault types. Algorithms created for this fault model, however, are considerably 
more complex and require more system resources than the ones developed for less strict fault models. For instance, many arbitrary fault model algorithms require that at most a third of the components of the system present any type of fault [Castro and Liskov 2002]. This is a reasonable requirement considering the strength of fault tolerance provided. Nonetheless, this implies that to achieve the same level of fault tolerance a system designer would have to use a third more machines than necessary for basic crash tolerance. Worse still, if one considers programming error as the more likely fault to be tolerated, no more than one third of the system is allowed to use the same implementation. This happens because if a single implementation were used in the entire system, a programming error in this implementation would generate faulty behavior in more than a third of the machines.

The choice of fault model can be considered a trade off between stronger fault tolerance and simpler implementation requiring fewer resources. In a sense, the gap between crash-tolerant fault models and arbitrary fault model is too large [Behrens et al. 2013]. One approach to reach a middle ground, focused on hardware faults, is the nonmalicious arbitrary fault model [Behrens et al. 2013]. This model assumes it is possible to detect and filter faults with a given probability, if these faults are not created with malicious intent, allowing the isolation and mapping of these faults to benign faults. In practice this means that it is possible to use a generic adapter that allows crash-tolerant algorithms to tolerate non-malicious arbitrary faults.

We believe it is possible to go further than detecting hardware faults, and detect other faults that are non-malicious, such as programming and configuration faults. By its non-malicious nature, the effects of these failures will be probabilistic detectable, and the system can be made tolerant to them. To this end we have as a first step modified an implementation of active replication [Schneider 1990] as a testbed for tolerating failures in a non-malicious arbitrary setting. We were successful in hardening the testbed against hardware failure and local data corruption [Barbieri and Vieira 2015].

In this paper we describe how we implemented a basic type of distributed validation, where a deviation from the expected algorithm behavior will make a process crash. We also experimentally evaluate the hardened testbed using a fault injection framework. Our results show that it is feasible to extend the concept of non-malicious failures beyond hardware failures. The main contributions of this paper are the description of the distributed validation used, the protocol to validate its effectiveness and evidence that this approach is sound. The paper starts by defining more precisely the non-malicious arbitrary fault model in Section 2 and how to implement algorithms for it in Section 3. Then, we describe our testbed and our proposed distributed validation in Section 4. We close the paper with an experimental evaluation of our implementation in Section 5.

\section{Fault Model}

Fault models abstract the properties a system must satisfy and how a distributed algorithm should tolerate faults. Two very common fault models in which distributed algorithms are designed are the crash-stop and crash-recovery models. In both models processes only fail by completely crashing. We can call these models benign because it is assumed that the tolerated failures will respect a (probably unknown) probability distribution. A stronger fault model that assumes more types of failures is the Byzantine or arbitrary 
model in which processes can deviate in any way from the algorithm specification.

In the arbitrary fault model it is impossible for processes to decide whether another process is behaving arbitrarily intentionally or not. This assumption covers virtually any type of failure a system might encounter. We refer to malicious faults when a process is behaving arbitrarily intentionally, through manipulation from a malicious agent. These type of faults do not follow a pre-defined probability distribution and can occur in response to measures taken to tolerate them.

Fault models range from weaker (more strict) to stronger (more general), as shown in Figure 1. The stronger the model, the more complex and difficult it is to implement an algorithm. When building a practical distributed system, it is desirable to adopt a fault model that better fits the system and satisfies its requirements for performance and types of faults it must tolerate. However, this is not always the case, since any distributed system that relies on actual computers is prone to arbitrary faults.

Distributed system designers desire to tolerate arbitrary faults, but would prefer a less performance intensive algorithm than a byzantine one [Bhatotia et al. 2010, Correia et al. 2012, Behrens et al. 2013]. While malicious faults are being tolerated using different techniques [Bhatotia et al. 2010, Correia et al. 2012], and based on the premise that any fault model can be hardened to tolerate some arbitrary faults, it is possible to harden the crash-recovery benign model to tolerate non-malicious arbitrary faults, thus achieving a fault model similar to the arbitrary fault model, but where malicious faults are not necessarily tolerated by the algorithm.

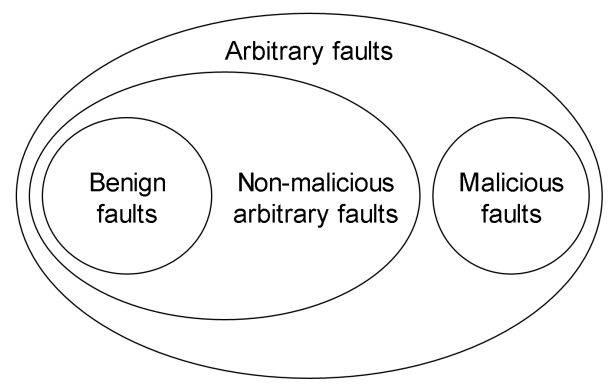

Figure 1. Relationship among fault classes

An algorithm for the non-malicious arbitrary fault model can be considered to be less complex than an classical arbitrary one because it does not tolerate malicious faults in its implementation. However, the implementation required to tolerate all nonmalicious arbitrary faults adds its own complexity to the algorithm. This fault model is more precisely described in terms of the two following properties [Behrens et al. 2013]:

No impersonation: the environment never creates valid messages, except for duplicates. This property assumes that only processes themselves are able to create valid messages, so malicious agents cannot interact with existing processes in a system.

No propagation: a process that is considered faulty, by either itself or by another process, cannot ever create a valid message. This property assumes that when the process has become faulty, it is not allowed to send any more messages, nor any correct process is allowed to accept messages from a faulty process. 
An algorithm for this fault model is expected to tolerate faults caused by data corruption, such as from persistent memory, main memory or network, in addition to benign faults [Bhatotia et al. 2010, Correia et al. 2012, Behrens et al. 2013].

\section{Tolerating Non-malicious Arbitrary Faults}

Non-malicious arbitrary fault types are present not only in any practical distributed system, but in any system that relies on computer components that can fail. These faults can be tolerated through error detection techniques, such as integrity checks and semantic checks. The techniques described below aim to detect data corruption, memory corruption, and in some cases programmer and operator mistakes. However, each approach mentioned has its overhead cost associated, for either performing repeated checks and recalculations, encrypting, or doubling memory requirements due to redundancy.

\subsection{Integrity checks}

Integrity checks verify data by saving at least one redundant copy that can be used to validate against the original data, such as checksums, duplicate states, timestamps or data size values. This approach is commonly used when reading and writing data from main memory, storage and peer-to-peer network message exchanges. We now describe in more detail these techniques:

Data and state redundancy : each process variable or stored data has a duplicate which can be validated against and used for backup. The duplicates must be always be kept in sync and checked for consistency on each read and write operation. This approach clearly uses a significant amount of additional memory and has an increased overhead for keeping both states in sync [Bhatotia et al. 2010, Correia et al. 2012, Chandra et al. 2007];

Checksums and hashes redundancy : the usage of encoded redundancy allows for future detection of undesired corruption. The most common type of redundancy is generating a checksum or hash of data and attaching it to the protocol messages prior to transmitting across the network or saving them in storage. Any read or write operation on data must recalculate the checksum and verify against the one attached to the message, adding a significant performance cost related to the checksum algorithm used [Bhatotia et al. 2010, Correia et al. 2012, Chandra et al. 2007];

Encoding and arithmetic codes : the usage of in-place encoding and decoding, like numerical properties of data, can be used to detect undesired corruption in each read and write operation. For instance, if numerical variables are multiplied by a prime number upon writing and divided by the same number when they are read back, the remainder should always be zero. This approach is considered to be very efficient performance-wise, but lacks coverage [Behrens et al. 2013].

\subsection{Semantic checks}

Semantic checks validate that after an operation has been applied on data, the newly obtained state is semantically correct according to the applied operation [Bhatotia et al. 2010]. For instance: after adding an element to a list, check if the element is in the list. This approach has the added benefit of testing the system against possible bugs, which was one scenario in the experiment found in [Chandra et al. 2007]. 


\section{Hardened Treplica}

\subsection{Treplica and Paxos}

Treplica [Vieira and Buzato 2008, 2010] is a Java framework that allows distributed applications to use Paxos as middleware to manage state replication through its state machine. Its implementation is close to the Multi-Paxos approach [Lamport 1998] with a few additional optimizations, like Fast Paxos support [Lamport 2006] and broadcast votes, where each learner agent receiving a majority of votes can commit the change immediately.

In Treplica, replicas can concurrently perform any Paxos role, such as coordinator, proposer, learner and acceptor. This is analogue to many practical middlewares implementing Paxos, and allows for greater flexibility in the amount of replicas and system configurations. Figure 2 illustrates Multi-Paxos algorithm messages exchanged during a common round in a consensus instance, highlighting the differences between theoretical Multi-Paxos and Treplica's implementation. The message roles are as follows:

Message \#1: Client proposal message sent to coordinator;

Message \#2: Proposal sent to acceptors for voting;

Message \#3: Acceptors vote on proposal;

Message \#4: Decision is broadcast to learners.

In Treplica, voting messages, labeled \#3 in the figure, are received by learners and the proposer as well, thus allowing learners to apply the state transition immediately. Also, the proposer can respond to the client as soon as receiving a majority of votes. Moreover, message \#4 is not necessary but is used to broadcast a decision if there is any message loss.

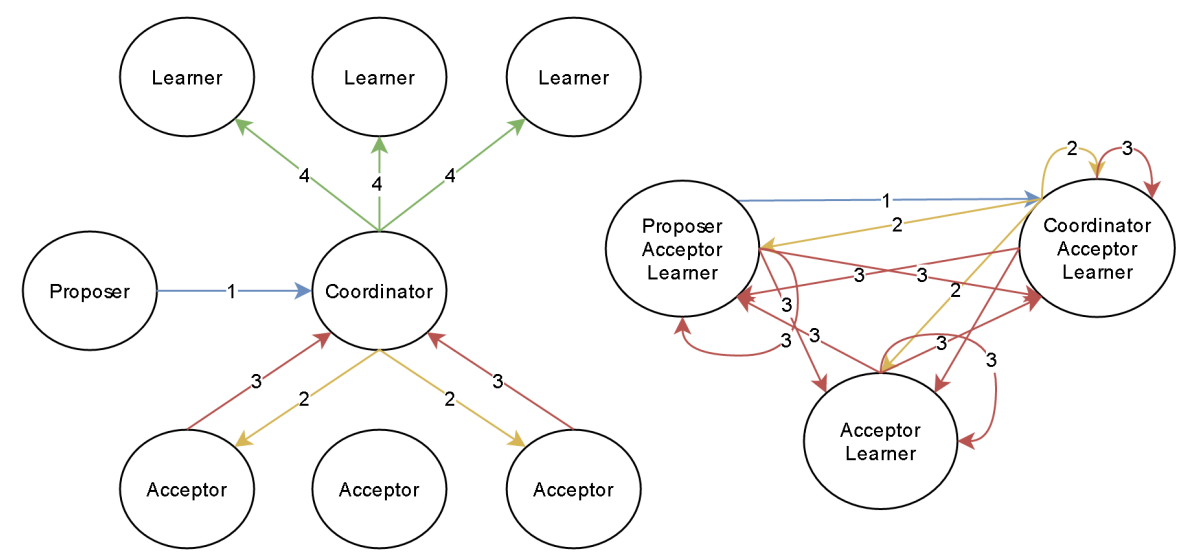

Figure 2. Multi-Paxos (left) and Multi-Paxos in Treplica (right)

Applications designed according to the Model-View-Controller standard can easily be modeled to use Treplica. We chose Treplica because its modular architecture allows for improvements to be easily coded and tested. Since it is designed to tolerate benign faults, upon analysis we validated that it is prone to non-malicious arbitrary faults we are interested in. Additionally, Treplica is object-oriented and makes use of immutable objects design, where an object is never changed after being instantiated. This allows for more efficient use of checksums. State transition semantic checks can also be easily coded by the application due to its integration with the state machine modeling. 


\subsection{Tolerating non-malicious arbitrary faults}

Our main approach to harden the benign crash-recovery fault model into the non-malicious arbitrary one is to employ fault detection techniques, as described in Section 3, to detect faults resulting from data corruption, while initially not worrying about how to recover from them. Moreover, we have created a distributed validation mechanism to try and detect deviations from the expected algorithm properties, that is, if the replicas state start to diverge. Upon detecting unexpected faults, our proposal is to abort the replica execution, preventing it from propagating corrupt data and further participating in the consensus algorithm. This strategy reduces the crash-recovery fault model to a simpler crash-stop one, but tolerating non-malicious arbitrary faults. This is similar to the approach taken by [Behrens et al. 2013], but adds the ability to detect protocol violations.

From the point of view of a benign fault model distributed system, most arbitrary faults behave as silent faults because their errors cannot be easily detected. For instance, if a user clicks a button to buy one book, but a replica processes that two books have been bought because bits got flipped along the way, then this is not an error from Paxos point of view, because the message was delivered consistently across all replicas. In order to effectively tolerate such silent faults, we employed the following techniques:

- Integrity checks, to address data corruption.

- State checks, to address state corruption.

- Semantic checks, to address programmer mistakes in the application.

- Distributed validation, to address data corruption, programmer or configuration faults.

Integrity, state and semantic checks were implemented based on the ideas described in Section 3. Details of this implementation can be found in [Barbieri and Vieira 2015], and are unfortunately omitted from this paper because of lack of space. Next, we describe in detail our proposal for distributed validation of replication algorithms.

\subsection{Distributed validation}

In a distributed system in which each replica has its independent state, although Paxos can guarantee state transitions are applied in the same order, it cannot guarantee that all replicas will have the same state in the presence of non-malicious arbitrary faults. A replica that experiences an arbitrary fault may have its state diverged from the others, while state transitions will continuously be applied on top of the corrupt state. This may allow the system to display incorrect data when the replica is queried by a client application. This divergence can happen by a corruption in the memory holding the code of the protocol or, more likely, be caused by programming or configuration fault. In order to completely satisfy the "No propagation" property of the fault model, this scenario must be covered by a validation mechanism.

A distributed validation mechanism allows replicas to validate their state upon receiving a network message containing a checksum or hash that is related to the state they currently are, thus detecting possible state divergences. A state of a replica comprises both the application state and the internal state of all Paxos agents. We developed a way to use the Paxos algorithm to perform this validation, thus having the algorithm extended with this mechanism. We attempt to detect state divergence between replicas by 
including the state checksum in the voting messages exchanged by Paxos in the accepting phase (Message \#3, see Section 4.1). Acceptors read the checksum from the application when creating the immutable voting messages and attach it to them. In Treplica, all replicas receive the voting messages, thus the learner module validates the local state upon receiving them using the attached checksum.

In order to minimize the performance impact and adapt the mechanism to Treplica's architecture, we decided to take an eventual and opportunistic validation approach. By defining a window of state counters in which the state checksum is updated, the replicas are able to eventually validate a state within the defined window. For instance, if the window value is 100 , then the state checksum will change only every one hundred state transitions have been applied. This makes it easier to synchronize all replicas in the same window, because Paxos, and many other distributed algorithms, does not impose a hard limit on the speed differences of the system processes. The only requirement is that a quorum be approximately synchronized, a single straggler process can be in a state arbitrarily behind the others.

In Figure 3, acceptors include their state checksum numbered \#14 when consensus instance of the state transition numbered \#15 is running. Both consensus instances $\# 14$ and \#15 are related to the same window, which is from state transition \#1 to state transition \#100, thus they carry the checksum generated in transition \#1. The learner validates state checksum numbered \#14 before committing the state transition numbered \#15. In this example, if any of the replicas have their state diverging within this window, it will be detectable only after transition \#100, where a new checksum will be included in exchanged messages. Without this window mechanism, replicas would rarely be able to validate received state checksums related to the same state count, because they apply the state transitions in an asynchronous way. Treplica packs a set of state transitions in the same Paxos instance, and replicas end up advancing rounds in different paces, resulting in the current window and backlog variables frequently getting discarded due to the state count advancing before having a chance to validate.

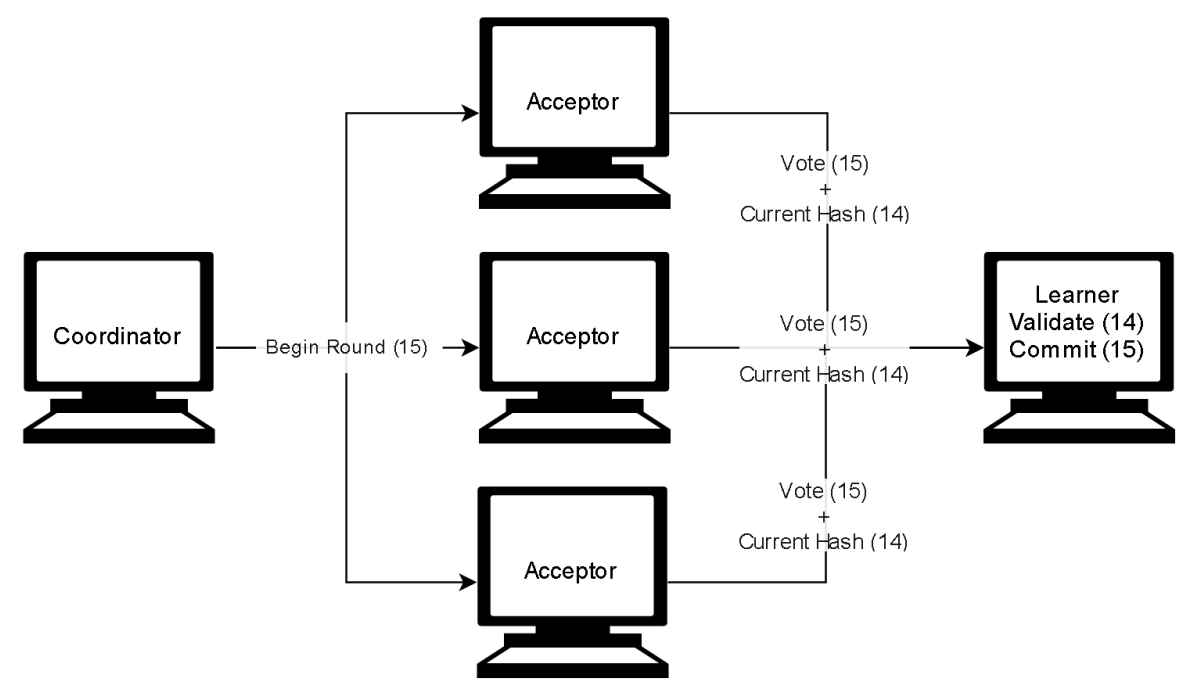

Figure 3. Distributed validation mechanism implemented in Treplica

We defined two variables where we store the received state checksums. One that 
is related to the current window, and a backlog one that is related to the next window to be processed. The next window is determined by the first received message that does not fit into the current window. Messages received that fit into the current window are validated immediately, while messages related to the subsequent registered window are stored for later validation. When a replica advances to the window that contains messages to be processed later, it moves all the stored messages to the current window variable, clears the backlog one and starts processing them.

Validation consists in comparing the validating replica's state checksum to the majority of received state checksums. As soon as the number of received common state checksums is the same as the number of replicas in the majority quorum in the system, the validating replica's state checksum is compared to this common state checksum. If the validating replica's state checksum is not the same as the majority, then the validating replica detects that it has diverged and aborts execution.

In Algorithm 1 it is shown the validation code that is performed by learners. Every state checksum received that is not related to the current window is saved for later processing. When a state checksum that matches the current window is received, it is saved in a structure responsible for storing state checksums indexed by replica unique identification numbers. This structure has a method getMostCommonChecksum() responsible for returning a list of the most common occurrence for the current window. If the size of this list matches the quorum size, then the learner validates its own checksum against that common checksum, which raises an exception if it diverges.

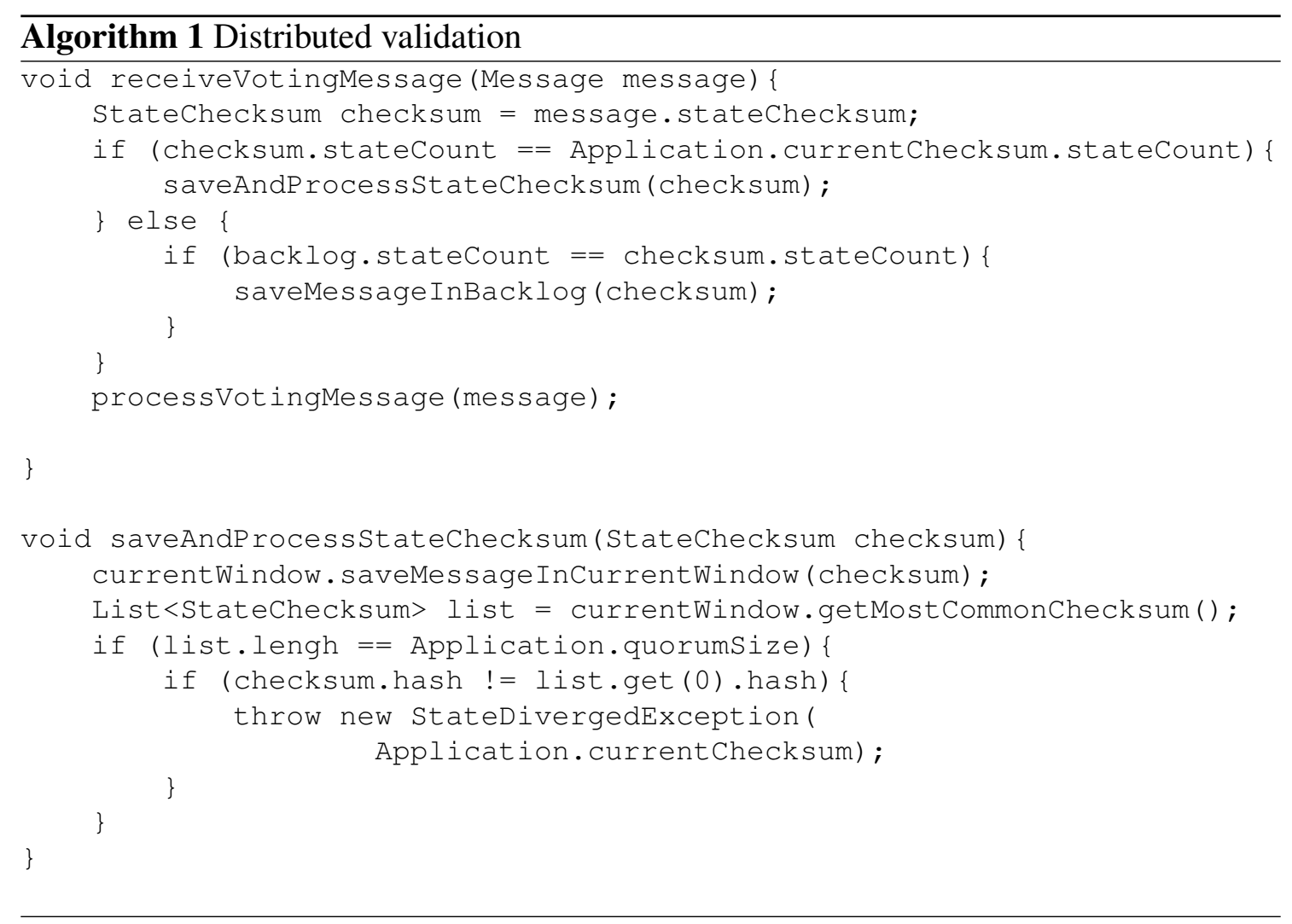

Expecting a majority is a way to avoid a correct process from terminating as it encounters the state from a corrupted process. If a majority of processes has the same 
state checksum and this value is different from the local state checksum, the process is certain to be one that diverged. We consider this mechanism to be eventual, since replicas may not participate in certain voting rounds. Moreover, this approach would fail in case a majority of replicas diverge in the same window. Such mechanism would still depend on the application being able to generate a checksum of its state or of certain data that is comparable to other replicas. The more precise this information, the more coverage this mechanism can achieve.

\section{Experimental Validation}

To evaluate the dependability of hardened Treplica, we injected randomly generated faults in a test system and analyzed the occurrence of errors. Our testing system consists in a set of replicas of a distributed application, implemented on top of Treplica. We have generated load to simulate user requests to each replica while injecting failures, and we have measured the error ratio of the hardened Treplica. In this section we describe our experimental setup, how faults were injected, the parameters of these fault injections, and the observed error rate.

\subsection{Test Setup}

In order to generate the load, the test system running an example application received requests which cause state transitions for five minutes. We created a manager application to run 50 instances of the same test, preparing and cleaning each replica instance resources used by each instance, such as storage folders and logs. When starting a test instance the manager application starts the replicas and starts the load generating application, which sends the requests. In case an error is detected, the application crashes or times out, then the manager application aborts the test execution, cleans the storage folders and logs the results to a separate folder indexed by the test instance count. Our request timeout was set to five minutes, in order to detect whether a failure made the system unable to continue processing requests.

We coded a simple application built on top of Treplica to perform tests: a hash table of strings. Client requests can add, remove and list elements in the hash. In this application, the application-defined state information implemented counts the number of elements in the hash. The semantic checks implemented for the "AddElement" operation validates if the added element is present in the hash, while for the "RemoveElement" operation it validates if the element is not present in the hash. Each request was always an "AddElement" operation, where the element consisted of a Long-type counter value converted to string value, appended by the replica identification number. When running tests with fault injections using this application, we always printed all the elements in the hash when the test finished without detected faults to get a record of the final state of the replica.

Instead of running Treplica on separate machines in a cluster, we opted to run five instances of the same application at the same time in a single machine. Each instance had a separate folder for individual storage, thus the replicas were independent. Running in the same machine makes it easier to overload the system, dropping and reordering messages, creating configurations not easily found in a system with low load. Curiously, we actually did run the tests in independent machines in a cluster to parallelize individual 
executions and cut total time required for the 50 runs of each experiment. Each node in the cluster had the following configuration: Intel Xeon E5-2665 $2.40 \mathrm{GHz}$ processor, 16 GB DDR3 1333 MHz RAM, 1 TB HDD, Linux CentOS 6, Java Development Kit (JDK) version 1.8.0 update 191. The specific amount of requests per second we chose was calibrated to overload it, creating messages reordering and loss. For this particular hardware we settled with $10000 \mathrm{Op} / \mathrm{s}$ per replica, for a total of $50000 \mathrm{Op} / \mathrm{s}$ total.

\subsection{Fault Injection using AspectJ}

To inject faults we used an aspect-oriented library known as AspectJ. This library allows us to change the behavior of any Java program without changing its main code. In Algorithm 2 it is shown an example injection, where in order to inject a fault in the operation of adding a string to a list, a method must be created to have its behavior overridden. The method in this case is "listAdd". Our injection code runs instead of the original code every time "listAdd" is invoked, we then use a local variable to decide whether we inject a fault that consists in running the original function with a different argument value, or if we allow the function to continue without faults. The idea is to "corrupt" the execution of the method as if its arguments were subject to bit flips or memory corruption.

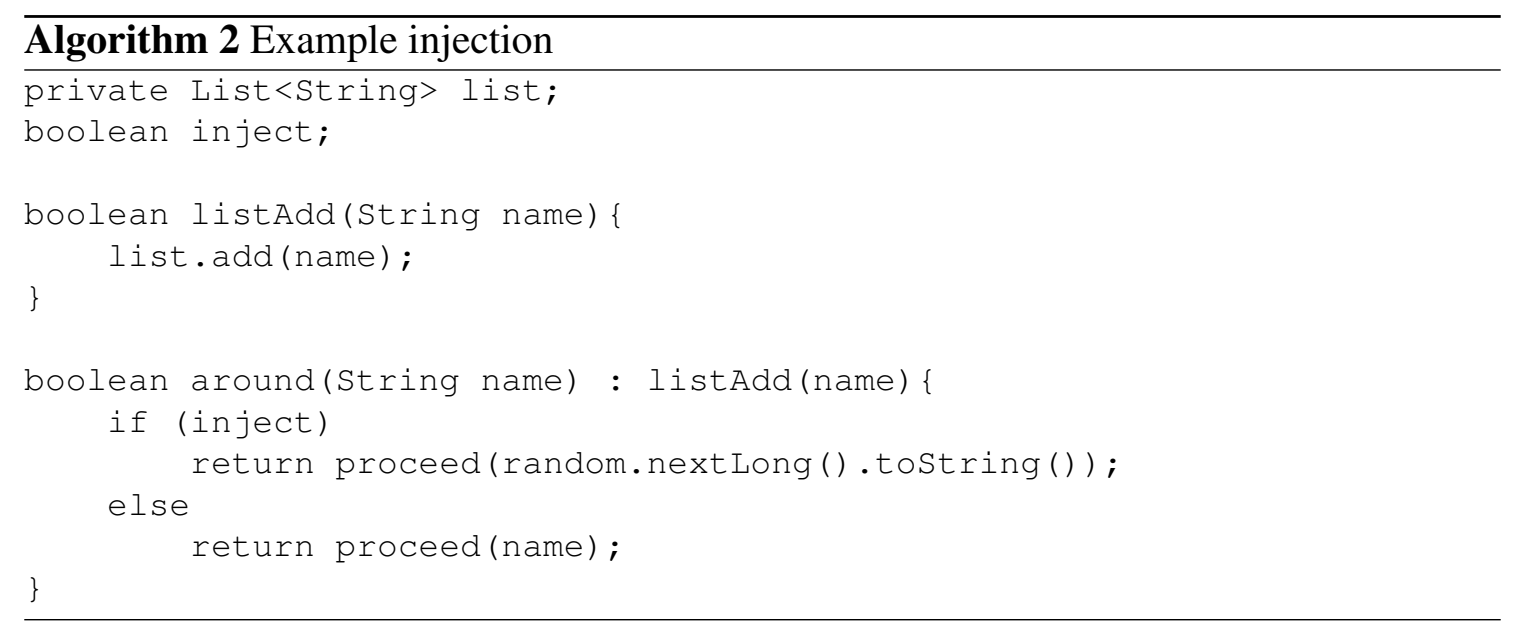

From this basic idea, we created a fault injection framework that reads a configuration file when Treplica is initialized and sets the fault injection conditions and modes according to a set of parameters. Whenever an aspect-marked method in the original code is executed, the aspect code is invoked. If the condition is not met, no code is injected and the original code is run. The two modes of operation implemented are:

Single timed injection mode: in this mode, a timer value is defined in the configuration file for each injection. Once the timer expires, the condition allows the fault to be injected. The fault is injected the next time the aspect is invoked. Once the fault is injected, the condition is permanently disabled. This mode is useful when we want to confirm that a single fault was tolerated or not;

Probability-based injection mode: in this mode, a probability value is defined in the configuration file for each injection. Whenever the aspect-marked code is executed, it randomly generates a probability value. If it is higher than the probability value defined, then the condition for fault injection is met and the fault is injected immediately. All subsequent executions of the aspect-marked code will check 
against the probability of injection, which may inject more fault occurrences. This mode is useful when we cannot guarantee that a single fault injection can cause an error to occur, because it may require specific conditions to be met. For example, a failure may require many consecutive messages to be lost, else the errors will be absorbed by the algorithm.

\subsubsection{Injection Scenarios}

With the framework in place, we choose three sets of specific faults to inject in the replicas: message faults, application faults and algorithm faults. Message injections corrupts data arriving or leaving the address space of the replica, such as network messages exchanged between replicas, log of operations saved and retrieved from secondary storage, and checkpoints, also saved and retrieved from secondary storage. Application injections corrupts the internal state of the application. Algorithm injections makes the algorithm behave differently from its specification.

For message injections, we used single timed injection mode to corrupt messages in transit. In network, storage and checkpoint messages, we change a random value in the message as soon as it is received from the network or recovered from storage, while retaining the checksum value on the hardened Treplica. For application injections, we used single timed injection mode to inject faults during state transition in the hash of strings application. We inject faults in the state by manipulating the state transition operation to either not add the elements or by changing their string values. For both message and application injections the fault was injected 10 seconds after the test started.

For algorithm injections, we used probability-based injection mode attempting to cause failures by breaking simple but very important algorithm mechanics, in order to cause replica states to diverge. Assuming the Paxos algorithm is keeping replica consistency, the faults injected are as follows:

Learner commits with no quorum: This injection causes the affected learner to have a probability of committing a proposal without requiring a quorum to do so. If the round does not succeed and a new value is proposed, the replica ends up with its state diverging from the others.

Acceptor forgets its previous votes: This injection causes the affected acceptor to have a probability of returning no previously registered votes for a given round. The coordinator, upon receiving this message, will assume that no previous value has been proposed, and will propose a new one instead, while the previous value may have been decided and committed by learners.

Coordinator forgets last proposals received: This injection causes the coordinator to have a probability of forgetting the previous proposals he receives from acceptors, thus choosing to start a new round with a new value while the previous value may have been decided and committed by learners.

We used $80 \%$ fault injection probability for the injection scenarios being tested. The Paxos algorithm is surprisingly resilient, even when the injected faults made it behave as if a programmer had made a mistake, the algorithm gives the correct answer most of the time. The high probability of fault injection coupled with the overload of the system 
Table 1. Message and application injection tests

\begin{tabular}{|l|l|l|l|l|l|}
\hline Test & Runs & $\begin{array}{l}\text { Fault } \\
\text { injections }\end{array}$ & $\begin{array}{l}\text { Fault } \\
\text { detections }\end{array}$ & Errors & Rate \\
\hline Message injections - Fault on a protocol message received & 100 & 100 & 100 & 0 & $100 \%$ \\
\hline Application injections - Fault when adding element & 100 & 100 & 100 & 0 & $100 \%$ \\
\hline
\end{tabular}

Table 2. Algorithm injection tests

\begin{tabular}{|l|l|l|l|l|l|}
\hline Test & Runs & $\begin{array}{l}\text { Fault } \\
\text { injections }\end{array}$ & $\begin{array}{l}\text { Fault } \\
\text { detections }\end{array}$ & Errors & Rate \\
\hline $\begin{array}{l}\text { Learner commits with no quorum } \\
\text { Single replica }\end{array}$ & 50 & 23500 & 45 & 1 & $98 \%$ \\
\hline $\begin{array}{l}\text { Acceptor forgets previous votes } \\
\text { Single replica }\end{array}$ & 50 & 3662900 & 0 & 0 & $100 \%$ \\
\hline $\begin{array}{l}\text { Learner commits with no quorum } \\
\text { All replicas }\end{array}$ & 50 & 194500 & 23 & 3 & $94 \%$ \\
\hline $\begin{array}{l}\text { Acceptor forgets previous votes } \\
\text { All replicas }\end{array}$ & 50 & 14584980 & 16 & 0 & $100 \%$ \\
\hline $\begin{array}{l}\text { Coordinator forgets received proposals } \\
\text { All replicas }\end{array}$ & 50 & 15000 & 47 & 2 & $96 \%$ \\
\hline
\end{tabular}

are required to create the scenarios in which the algorithm exhibit an error. With the selected parameters, we had a high probability at least one replica to commit an undecided proposal in each test run, causing a state divergence.

However, to assess the behavior of hardened Treplica with more faults we also ran tests where we performed Paxos injections in all replicas. Those test runs are labeled "All replicas" in the table, whereas the "Single replica" labels refer to injections performed in a single replica. We noticed that the test runs where we performed injections in all replicas were more likely to result in all replicas diverging. Additionally, we analyzed the possibility of the fault injected causing failures only to the replica which it was injected, or if errors propagated. In all our test runs, at least one execution had error propagation.

\subsection{Injection Test Results}

Table 1 lists the error rates for message and application fault injection tests in hardened Treplica. These faults model hardware faults that happen locally in a replica. For each run, a fault was injected and it was detected by the integrity, state or semantic checks. We confirmed the detection of all fault occurrences either through logging or replicas aborting their executions upon detection.

For algorithm injections, the error rate is shown in Table 2. For the test runs where faults were not detected, there were cases where there were no errors, thus there was no state divergence between any of the replicas. We confirmed such scenario by comparing all the elements printed by each replica at the end of a test run. The runs where there were errors, however, a majority of replicas diverged to different states, rendering the distributed validation incapable of detecting any divergence for the rest of the test run. These cases could also be confirmed by comparing the printed elements. We consider these occurrences to be beyond our technique's coverage capability at this moment.

Hardened Treplica was able to detect faults in all the fault injection scenarios, except for when all replicas got their state diverged in algorithm injection scenarios. The 
Table 3. Non-malicious fault class coverage

\begin{tabular}{|l|l|l|l|l|}
\hline Fault & Detected? & Fault model mapped to & Can be propagated? & Rate \\
\hline $\begin{array}{l}\text { Message injections } \\
\text { (network) }\end{array}$ & Yes & $\begin{array}{l}\text { Benign crash-recovery } \\
\text { (single message loss) }\end{array}$ & No & $100 \%$ \\
\hline $\begin{array}{l}\text { Message injections } \\
\text { (stable storage) }\end{array}$ & Yes & $\begin{array}{l}\text { Benign crash-stop } \\
\text { (replica unavailable) }\end{array}$ & No & $100 \%$ \\
\hline $\begin{array}{l}\text { Application injections } \\
\text { (memory corruption) }\end{array}$ & $\begin{array}{l}\text { Yes, but requires } \\
\text { application support }\end{array}$ & $\begin{array}{l}\text { Benign crash-recovery } \\
\text { (replica restarted) }\end{array}$ & Not applicable & $100 \%$ \\
\hline $\begin{array}{l}\text { Application injections } \\
\text { (bugs) }\end{array}$ & $\begin{array}{l}\text { Yes, but requires } \\
\text { application support }\end{array}$ & $\begin{array}{l}\text { Benign crash-stop } \\
\text { (replica unavailable) }\end{array}$ & Not applicable & $100 \%$ \\
\hline Paxos injections & Mostly & $\begin{array}{l}\text { Benign crash-stop } \\
\text { (replica unavailable) }\end{array}$ & Yes & $97,6 \%$ \\
\hline
\end{tabular}

only scenario we injected faults and we could not detect the resulting errors was the case of a majority of replicas (five replicas in our test suite), diverging while in the same state count window. We consider the following conditions are needed for this to happen:

- There must be several consecutive messages lost while the fault is injected. We validated that without message loss, there could not be any failure.

- The majority of replicas needs to diverge within the same state validation window (see Section 4.3). The divergence of individual replicas can be easily detected once the validation window changes and updates the state checksum, if there is still a majority of correct replicas.

We summarize our test suite results and analysis in Table 3, where we check for each injection scenario we tested:

- If a potential error from the fault injected was successfully detected and which conditions were necessary for this detection;

- To which fault model the fault occurrence can be mapped to and what other replicas observe of the faulty replica;

- If the error can be propagated and disrupt other replicas;

- Our coverage rate for the given fault.

Upon detecting a failure, we abort the replica execution. Our current solution results in a crash-stop non-malicious arbitrary fault model instead of an ideal crash-recovery non-malicious arbitrary fault model. We consider the non-malicious arbitrary crash-stop fault model to be more resilient than the original benign crash-recovery implementation. If a benign fault occurs, the system is able to recover itself and continue, but if an error from a non-malicious arbitrary fault is detected and is non-recoverable, we abort the replica execution, preventing any propagation of erroneous behavior.

\section{Conclusion}

In this paper we described the non-malicious arbitrary fault model and how we adapted the implementation of active replication found in the Treplica framework to use it. We proposed a novel distributed validation mechanism that expands the scope of non-malicious arbitrary faults tolerated. Our experimental evaluation has shown a very good coverage of protocol deviation faults, reaching $97,6 \%$ in the sum of all tests.

These results are particularly good, because here we report our very first attempt to detect deviations from the distributed algorithm. The idea is very simple and is prone 
to fail to detect multiple faults if they happen too fast. We believe this is a promising approach and we intend to create a more robust distributed validation mechanism.

\section{Acknowledgments}

This research was partially funded by the Brazilian Coordenação de Aperfeiçoamento de Pessoal de Nível Superior (CAPES) under the Pró-Equipamentos program (Edital 25/2011).

\section{References}

Barbieri, R. R. and Vieira, G. M. D. (2015). Hardened paxos through consistency validation. In SBESC '15: Proceedings of the V Brazilian Symposium on Computing Systems Engineering, SBESC '15, pages 13-18, Foz do Iguaçu, Brazil. IEEE Computer Society.

Behrens, D., Weigert, S., and Fetzer, C. (2013). Automatically tolerating arbitrary faults in non-malicious settings. In Dependable Computing (LADC), 2013 Sixth LatinAmerican Symposium on, pages 114-123.

Bhatotia, P., Wieder, A., Rodrigues, R., Junqueira, F., and Reed, B. (2010). Reliable datacenter scale computations. In Proceedings of the 4th International Workshop on Large Scale Distributed Systems and Middleware, LADIS '10, pages 1-6, New York, NY, USA. ACM.

Castro, M. and Liskov, B. (2002). Practical byzantine fault tolerance and proactive recovery. ACM Trans. Comput. Syst., 20(4):398-461.

Chandra, T. D., Griesemer, R., and Redstone, J. (2007). Paxos made live: An engineering perspective. In Proceedings of the Twenty-sixth Annual ACM Symposium on Principles of Distributed Computing, PODC '07, pages 398-407, New York, NY, USA. ACM.

Correia, M., Ferro, D. G., Junqueira, F. P., and Serafini, M. (2012). Practical hardening of crash-tolerant systems. In USENIX Annual Technical Conference, pages 453-466.

Lamport, L. (1998). The part-time parliament. ACM Trans. Comput. Syst., 16(2):133169.

Lamport, L. (2006). Fast paxos. Distributed Computing, 19(2):79-103.

Lamport, L., Shostak, R., and Pease, M. (1982). The byzantine generals problem. ACM Trans. Program. Lang. Syst., 4(3):382-401.

Schneider, F. B. (1990). Implementing fault-tolerant services using the state machine approach: A tutorial. ACM Comput. Surv., 22(4):299-319.

Vieira, G. M. D. and Buzato, L. E. (2008). Treplica: ubiquitous replication. In SBRC'08: Proc. of the 26th Brazilian Symposium on Computer Networks and Distributed Systems.

Vieira, G. M. D. and Buzato, L. E. (2010). Implementation of an object-oriented specification for active replication using consensus. Technical Report IC-10-26, Institute of Computing, University of Campinas. 